\title{
Research Article \\ On the Oscillation of Second-Order Neutral Delay Differential Equations
}

\author{
Zhenlai Han, ${ }^{1,2}$ Tongxing Li, ${ }^{1}$ Shurong Sun, ${ }^{1,3}$ \\ and Weisong Chen ${ }^{1}$
}

${ }^{1}$ School of Science, University of Jinan, Jinan, Shandong 250022, China

${ }^{2}$ School of Control Science and Engineering, Shandong University, Jinan, Shandong 250061, China

${ }^{3}$ Department of Mathematics and Statistics, Missouri University of Science and Technology, Rolla, MO 65409-0020, China

Correspondence should be addressed to Zhenlai Han, hanzhenlai@163.com

Received 8 October 2009; Accepted 10 January 2010

Academic Editor: Toka Diagana

Copyright (C) 2010 Zhenlai Han et al. This is an open access article distributed under the Creative Commons Attribution License, which permits unrestricted use, distribution, and reproduction in any medium, provided the original work is properly cited.

Some new oscillation criteria for the second-order neutral delay differential equation $\left(r(t) z^{\prime}(t)\right)^{\prime}+$ $q(t) x(\sigma(t))=0, t \geq t_{0}$ are established, where $\int_{t_{0}}^{\infty}(1 / r(t)) d t=\infty, z(t)=x(t)+p(t) x(\tau(t)), 0 \leq p(t) \leq$ $p_{0}<\infty, q(t)>0$. These oscillation criteria extend and improve some known results. An example is considered to illustrate the main results.

\section{Introduction}

Neutral differential equations find numerous applications in natural science and technology. For instance, they are frequently used for the study of distributed networks containing lossless transmission lines; see Hale [1]. In recent years, many studies have been made on the oscillatory behavior of solutions of neutral delay differential equations, and we refer to the recent papers [2-23] and the references cited therein.

This paper is concerned with the oscillatory behavior of the second-order neutral delay differential equation

$$
\left(r(t) z^{\prime}(t)\right)^{\prime}+q(t) x(\sigma(t))=0, \quad t \geq t_{0}
$$

where $z(t)=x(t)+p(t) x(\tau(t))$. 
In what follows we assume that

$\left(I_{1}\right) p, q \in C\left(\left[t_{0}, \infty\right), R\right), 0 \leq p(t) \leq p_{0}<\infty, q(t)>0$,

$\left(I_{2}\right) r \in C\left(\left[t_{0}, \infty\right), R\right), r(t)>0, \int_{t_{0}}^{\infty}(1 / r(t)) \mathrm{d} t=\infty$,

$\left(I_{3}\right) \tau, \sigma \in C\left(\left[t_{0}, \infty\right), R\right), \tau(t) \leq t, \sigma(t) \leq t, \tau^{\prime}(t)=\tau_{0}>0, \sigma^{\prime}(t)>0, \lim _{t \rightarrow \infty} \tau(t)=$ $\lim _{t \rightarrow \infty} \sigma(t)=\infty, \tau(\sigma(t))=\sigma(\tau(t))$, where $\tau_{0}$ is a constant.

Some known results are established for (1.1) under the condition $0 \leq p(t)<1$. Grammatikopoulos et al. [6] obtained that if $0 \leq p(t) \leq 1, q(t) \geq 0$ and, $\int_{t_{0}}^{\infty} q(s)[1-p(s-\sigma)] \mathrm{d} s=$ $\infty$, then the second-order neutral delay differential equation

$$
[y(t)+p(t) y(t-\tau)]^{\prime \prime}+q(t) y(t-\sigma)=0
$$

oscillates. In [13], by employing Riccati technique and averaging functions method, Ruan established some general oscillation criteria for second-order neutral delay differential equation

$$
\left[a(t)(x(t)+p(t) x(t-\tau))^{\prime}\right]^{\prime}+q(t) f(x(t-\sigma))=0
$$

$\mathrm{Xu}$ and Meng [18] as well as Zhuang and Li [23] studied the oscillation of the second-order neutral delay differential equation

$$
\left[r(t)(y(t)+p(t) y(\tau(t)))^{\prime}\right]^{\prime}+\sum_{i=1}^{n} q_{i}(t) f_{i}\left(y\left(\sigma_{i}(t)\right)\right)=0
$$

Motivated by [11], we will further the investigation and offer some more general new oscillation criteria for (1.1), by employing a class of function $Y$, operator $T$, and the Riccati technique and averaging technique.

Following [11], we say that a function $\phi=\phi(t, s, l)$ belongs to the function class $Y$, denoted by $\phi \in Y$ if $\phi \in C(E, R)$, where $E=\left\{(t, s, l): t_{0} \leq l \leq s \leq t<\infty\right\}$, which satisfies $\phi(t, t, l)=0, \phi(t, l, l)=0$, and $\phi(t, s, l)>0$, for $l<s<t$, and has the partial derivative $\partial \phi / \partial s$ on $E$ such that $\partial \phi / \partial s$ is locally integrable with respect to $s$ in $E$. By choosing the special function $\phi$, it is possible to derive several oscillation criteria for a wide range of differential equations.

Define the operator $T[\because l, t]$ by

$$
T[g ; l, t]=\int_{l}^{t} \phi(t, s, l) g(s) \mathrm{d} s
$$

for $t \geq s \geq l \geq t_{0}$ and $g \in C^{1}\left[t_{0}, \infty\right)$. The function $\varphi=\varphi(t, s, l)$ is defined by

$$
\frac{\partial \phi(t, s, l)}{\partial s}=\varphi(t, s, l) \phi(t, s, l) .
$$


It is easy to see that $T[\because l, t]$ is a linear operator and that it satisfies

$$
T\left[g^{\prime} ; l, t\right]=-T[g \varphi ; l, t], \quad \text { for } g(s) \in C^{1}\left[t_{0}, \infty\right)
$$

\section{Main Results}

In this section, we give some new oscillation criteria for (1.1). We start with the following oscillation criteria.

Theorem 2.1. If

$$
\int_{t_{0}}^{\infty} Q(t) d t=\infty
$$

where $Q(t):=\min \{q(t), q(\tau(t))\}$, then (1.1) oscillates.

Proof. Let $x$ be a nonoscillatory solution of (1.1). Then there exists $t_{1} \geq t_{0}$ such that $x(t) \neq 0$, for all $t \geq t_{1}$. Without loss of generality, we assume that $x(t)>0, x(\tau(t))>0$, and $x(\sigma(t))>0$, for all $t \geq t_{1}$. From (1.1), we have

$$
\left(r(t) z^{\prime}(t)\right)^{\prime}=-q(t) x(\sigma(t))<0, \quad t \geq t_{1} .
$$

Therefore $r(t) z^{\prime}(t)$ is a decreasing function. We claim that $z^{\prime}(t)>0$ for $t \geq t_{1}$. Otherwise, there exists $t_{2} \geq t_{1}$ such that $z^{\prime}\left(t_{2}\right)<0$. Then from (2.2) we obtain

$$
r(t) z^{\prime}(t) \leq r\left(t_{2}\right) z^{\prime}\left(t_{2}\right), \quad t \geq t_{2}
$$

and hence,

$$
z(t) \leq z\left(t_{2}\right)-\left[-r\left(t_{2}\right) z^{\prime}\left(t_{2}\right)\right] \int_{t_{2}}^{t} \frac{\mathrm{d} s}{r(s)}
$$

Taking $t \rightarrow \infty$, we get $z(t) \rightarrow-\infty, t \rightarrow \infty$. This contradiction proves that $z^{\prime}(t)>0$ for $t \geq t_{1}$. Using definition of $z(t)$ and applying (1.1), we get for sufficiently large $t$

$$
\left(r(t) z^{\prime}(t)\right)^{\prime}+q(t) x(\sigma(t))+p_{0} q(\tau(t)) x(\sigma(\tau(t)))+\frac{p_{0}}{\tau^{\prime}(t)}\left(r(\tau(t)) z^{\prime}(\tau(t))\right)^{\prime}=0,
$$

and thus,

$$
\left(r(t) z^{\prime}(t)\right)^{\prime}+Q(t) z(\sigma(t))+\frac{p_{0}}{\tau^{\prime}(t)}\left(r(\tau(t)) z^{\prime}(\tau(t))\right)^{\prime} \leq 0
$$


Integrating (2.6) from $t_{3}\left(\geq t_{1}\right)$ to $t$, we obtain

$$
\int_{t_{3}}^{t}\left(r(s) z^{\prime}(s)\right)^{\prime} \mathrm{d} s+\int_{t_{3}}^{t} Q(s) z(\sigma(s)) \mathrm{d} s+p_{0} \int_{t_{3}}^{t} \frac{1}{\tau^{\prime}(s)}\left(r(\tau(s)) z^{\prime}(\tau(s))\right)^{\prime} \mathrm{d} s \leq 0 .
$$

Noting that $\tau^{\prime}(t)=\tau_{0}>0$, we have

$$
\begin{aligned}
\int_{t_{3}}^{t} Q(s) z(\sigma(s)) \mathrm{d} s & \leq-\int_{t_{3}}^{t}\left(r(s) z^{\prime}(s)\right)^{\prime} \mathrm{d} s-p_{0} \int_{t_{3}}^{t} \frac{1}{\left(\tau^{\prime}(s)\right)^{2}}\left(r(\tau(s)) z^{\prime}(\tau(s))\right)^{\prime} \mathrm{d}(\tau(s)) \\
& =-\int_{t_{3}}^{t}\left(r(s) z^{\prime}(s)\right)^{\prime} \mathrm{d} s-\frac{p_{0}}{\tau_{0}^{2}} \int_{\tau\left(t_{3}\right)}^{\tau(t)}\left(r(u) z^{\prime}(u)\right)^{\prime} \mathrm{d} u \\
& =r\left(t_{3}\right) z^{\prime}\left(t_{3}\right)-r(t) z^{\prime}(t)+\frac{p_{0}}{\tau_{0}^{2}} r\left(\tau\left(t_{3}\right)\right) z^{\prime}\left(\tau\left(t_{3}\right)\right)-\frac{p_{0}}{\tau_{0}^{2}} r(\tau(t)) z^{\prime}(\tau(t)) .
\end{aligned}
$$

Since $z^{\prime}(t)>0$ for $t \geq t_{1}$, we can find a constant $c>0$ such that $z(\sigma(t)) \geq c$ for $t \geq t_{3} \geq t_{1}$. Then from (2.8) and the fact that $r(t) z^{\prime}(t)$ is eventually decreasing, we have

$$
\int_{t_{3}}^{\infty} Q(t) \mathrm{d} t<\infty
$$

which is a contradiction to (2.1). This completes the proof.

Theorem 2.2. Assume that $\sigma(t) \leq \tau(t)$, and there exist functions $\phi \in Y$ and $k \in C^{1}\left(\left[t_{0}, \infty\right), R^{+}\right)$ such that

$$
\limsup _{t \rightarrow \infty} T\left[k(s) Q(s)-\frac{\left(1+\left(p_{0} / \tau_{0}\right)\right)\left(\varphi+\left(k^{\prime}(s) / k(s)\right)\right)^{2}}{4} \frac{r(\sigma(s)) k(s)}{\sigma^{\prime}(s)} ; l, t\right]>0
$$

where $Q(t)$ is defined as in Theorem 2.1, the operator $T$ is defined by (1.5), and $\varphi=\varphi(t, s, l)$ is defined by (1.6). Then every solution $x$ of (1.1) is oscillatory.

Proof. Let $x$ be a nonoscillatory solution of (1.1). Then there exists $t_{1} \geq t_{0}$ such that $x(t) \neq 0$ for all $t \geq t_{1}$. Without loss of generality, we assume that $x(t)>0, x(\tau(t))>0$, and $x(\sigma(t))>0$, for all $t \geq t_{1}$. Define

$$
\omega(t)=k(t) \frac{r(t) z^{\prime}(t)}{z(\sigma(t))}, \quad t \geq t_{1}
$$

Then $w(t)>0$ and

$$
\omega^{\prime}(t)=k^{\prime}(t) \frac{r(t) z^{\prime}(t)}{z(\sigma(t))}+k(t) \frac{\left(r(t) z^{\prime}(t)\right)^{\prime} z(\sigma(t))-r(t) z^{\prime}(t) z^{\prime}(\sigma(t)) \sigma^{\prime}(t)}{z^{2}(\sigma(t))} .
$$


By (2.2) and the fact $z^{\prime}(t)>0$, we get

$$
\frac{z^{\prime}(\sigma(t))}{z^{\prime}(t)} \geq \frac{r(t)}{r(\sigma(t))}
$$

From (2.11), (2.12), and (2.13), we have

$$
\omega^{\prime}(t) \leq k(t) \frac{\left(r(t) z^{\prime}(t)\right)^{\prime}}{z(\sigma(t))}+\frac{k^{\prime}(t)}{k(t)} \omega(t)-\frac{\sigma^{\prime}(t)}{r(\sigma(t)) k(t)} \omega^{2}(t) .
$$

Similarly, define

$$
v(t)=k(t) \frac{r(\tau(t)) z^{\prime}(\tau(t))}{z(\sigma(t))}, \quad t \geq t_{1} .
$$

Then $v(t)>0$ and

$$
v^{\prime}(t)=k^{\prime}(t) \frac{r(\tau(t)) z^{\prime}(\tau(t))}{z(\sigma(t))}+k(t) \frac{\left(r(\tau(t)) z^{\prime}(\tau(t))\right)^{\prime} z(\sigma(t))-r(\tau(t)) z^{\prime}(\tau(t)) z^{\prime}(\sigma(t)) \sigma^{\prime}(t)}{z^{2}(\sigma(t))}
$$

By (2.2) and the facting $z^{\prime}(t)>0$, noting that $\sigma(t) \leq \tau(t)$, we get

$$
\frac{z^{\prime}(\sigma(t))}{z^{\prime}(\tau(t))} \geq \frac{r(\tau(t))}{r(\sigma(t))}
$$

From (2.15), (2.16), and (2.17), we have

$$
v^{\prime}(t) \leq k(t) \frac{\left(r(\tau(t)) z^{\prime}(\tau(t))\right)^{\prime}}{z(\sigma(t))}+\frac{k^{\prime}(t)}{k(t)} v(t)-\frac{\sigma^{\prime}(t)}{r(\sigma(t)) k(t)} v^{2}(t)
$$

Therefore, from (2.14) and (2.18), we get

$$
\begin{aligned}
\omega^{\prime}(t)+\frac{p_{0}}{\tau_{0}} v^{\prime}(t) \leq & k(t) \frac{\left(r(t) z^{\prime}(t)\right)^{\prime}}{z(\sigma(t))}+\frac{p_{0}}{\tau_{0}} k(t) \frac{\left(r(\tau(t)) z^{\prime}(\tau(t))\right)^{\prime}}{z(\sigma(t))} \\
& +\frac{k^{\prime}(t)}{k(t)} \omega(t)-\frac{\sigma^{\prime}(t)}{r(\sigma(t)) k(t)} \omega^{2}(t)+\frac{p_{0}}{\tau_{0}} \frac{k^{\prime}(t)}{k(t)} v(t)-\frac{p_{0}}{\tau_{0}} \frac{\sigma^{\prime}(t)}{r(\sigma(t)) k(t)} v^{2}(t) .
\end{aligned}
$$

From (2.6), we obtain

$$
\begin{aligned}
\omega^{\prime}(t)+\frac{p_{0}}{\tau_{0}} v^{\prime}(t) \leq & -k(t) Q(t)+\frac{k^{\prime}(t)}{k(t)} \omega(t)-\frac{\sigma^{\prime}(t)}{r(\sigma(t)) k(t)} \omega^{2}(t) \\
& +\frac{p_{0}}{\tau_{0}} \frac{k^{\prime}(t)}{k(t)} v(t)-\frac{p_{0}}{\tau_{0}} \frac{\sigma^{\prime}(t)}{r(\sigma(t)) k(t)} v^{2}(t) .
\end{aligned}
$$


Applying $T[\because ; l, t]$ to $(2.20)$, we get

$$
\begin{aligned}
& T\left[\omega^{\prime}(s)+\frac{p_{0}}{\tau_{0}} v^{\prime}(s) ; l, t\right] \\
& \quad \leq T\left[-k(s) Q(s)+\frac{k^{\prime}(s)}{k(s)} \omega(s)-\frac{\sigma^{\prime}(s)}{r(\sigma(s)) k(s)} \omega^{2}(s)+\frac{p_{0}}{\tau_{0}} \frac{k^{\prime}(s)}{k(s)} v(s)-\frac{p_{0}}{\tau_{0}} \frac{\sigma^{\prime}(s)}{r(\sigma(s)) k(s)} v^{2}(s) ; l, t\right] .
\end{aligned}
$$

By (1.7) and the above inequality, we obtain

$$
\begin{aligned}
& T[k(s) Q(s) ; l, t] \\
& \leq T\left[\left(\varphi+\frac{k^{\prime}(s)}{k(s)}\right) \omega(s)-\frac{\sigma^{\prime}(s)}{r(\sigma(s)) k(s)} \omega^{2}(s)+\frac{p_{0}}{\tau_{0}}\left(\varphi+\frac{k^{\prime}(s)}{k(s)}\right) v(s)-\frac{p_{0}}{\tau_{0}} \frac{\sigma^{\prime}(s)}{r(\sigma(s)) k(s)} v^{2}(s) ; l, t\right] .
\end{aligned}
$$

Hence, from (2.22) we have

$$
T[k(s) Q(s) ; l, t] \leq T\left[\left(\frac{\left(\varphi+\left(k^{\prime}(s) / k(s)\right)\right)^{2}}{4}+\frac{\left(p_{0} / \tau_{0}\right)\left(\varphi+\left(k^{\prime}(s) / k(s)\right)\right)^{2}}{4}\right) \frac{r(\sigma(s)) k(s)}{\sigma^{\prime}(s)} ; l, t\right],
$$

that is,

$$
T\left[k(s) Q(s)-\frac{\left(1+\left(p_{0} / \tau_{0}\right)\right)\left(\varphi+\left(k^{\prime}(s) / k(s)\right)\right)^{2}}{4} \frac{r(\sigma(s)) k(s)}{\sigma^{\prime}(s)} ; l, t\right] \leq 0 .
$$

Taking the super limit in the above inequality, we get

$$
\limsup _{t \rightarrow \infty} T\left[k(s) Q(s)-\frac{\left(1+\left(p_{0} / \tau_{0}\right)\right)\left(\varphi+\left(k^{\prime}(s) / k(s)\right)\right)^{2}}{4} \frac{r(\sigma(s)) k(s)}{\sigma^{\prime}(s)} ; l, t\right] \leq 0
$$

which contradicts (2.10). This completes the proof.

Remark 2.3. With the different choice of $k$ and $\phi$, Theorem 2.2 can be stated with different conditions for oscillation of (1.1). For example, if we choose $\phi(t, s, l)=\rho(s)(t-s)^{\sigma}(s-l)^{\mu}$ for $\sigma>1 / 2, \mu>1 / 2, \rho \in C^{1}\left(\left[t_{0}, \infty\right),(0, \infty)\right)$, then

$$
\varphi(t, s, l)=\frac{\rho^{\prime}(s)}{\rho(s)}+\frac{\mu t-(\sigma+\mu) s+\sigma l}{(t-s)(s-l)}
$$

By Theorem 2.2 we can obtain the oscillation criterion for (1.1), the details are left to the reader. 
For an application, we give the following example to illustrate the main results.

Example 2.4. Consider the following equation:

$$
(x(t)+2 x(t-\pi))^{\prime \prime}+x(t-\pi)=0, \quad t \geq t_{0} .
$$

Let $r(t)=1, p(t)=2, q(t)=1$, and $\tau(t)=\sigma(t)=t-\pi$, then by Theorem 2.1 every solution of (2.27) oscillates; for example, $x(t)=\sin t$ is an oscillatory solution of (2.27).

Remark 2.5. The recent results cannot be applied in (2.27) since $p(t)=2>1$; so our results are new ones.

\section{Acknowledgments}

This research is supported by the Natural Science Foundation of China $(60774004,60904024)$, China Postdoctoral Science Foundation Funded Project (20080441126, 200902564), Shandong Postdoctoral Funded Project (200802018) and the Natural Scientific Foundation of Shandong Province (Y2008A28, ZR2009AL003), also supported by University of Jinan Research Funds for Doctors (XBS0843).

\section{References}

[1] J. Hale, Theory of Functional Differential Equations, Springer, New York, NY, USA, 2nd edition, 1977, Applied Mathematical Sciences.

[2] R. P. Agarwal and S. R. Grace, "Oscillation theorems for certain neutral functional-differential equations," Computers \& Mathematics with Applications, vol. 38, no. 11-12, pp. 1-11, 1999.

[3] L. Berezansky, J. Diblik, and Z. Šmarda, "On connection between second-order delay differential equations and integrodifferential equations with delay," Advances in Difference Equations, vol. 2010, Article ID 143298, 8 pages, 2010.

[4] J. Džurina and I. P. Stavroulakis, "Oscillation criteria for second-order delay differential equations," Applied Mathematics and Computation, vol. 140, no. 2-3, pp. 445-453, 2003.

[5] S. R. Grace, "Oscillation theorems for nonlinear differential equations of second order," Journal of Mathematical Analysis and Applications, vol. 171, no. 1, pp. 220-241, 1992.

[6] M. K. Grammatikopoulos, G. Ladas, and A. Meimaridou, "Oscillations of second order neutral delay differential equations," Radovi Matematički, vol. 1, no. 2, pp. 267-274, 1985.

[7] Z. Han, T. Li, S. Sun, and Y. Sun, "Remarks on the paper [Appl. Math. Comput. 207 (2009) 388-396]," Applied Mathematics and Computation, vol. 215, no. 11, pp. 3998-4007, 2010.

[8] B. Karpuz, J. V. Manojlović, Ö. Öcalan, and Y. Shoukaku, "Oscillation criteria for a class of secondorder neutral delay differential equations," Applied Mathematics and Computation, vol. 210, no. 2, pp. 303-312, 2009.

[9] H.-J. Li and C.-C. Yeh, "Oscillation criteria for second-order neutral delay difference equations," Computers \& Mathematics with Applications, vol. 36, no. 10-12, pp. 123-132, 1998.

[10] X. Lin and X. H. Tang, "Oscillation of solutions of neutral differential equations with a superlinear neutral term," Applied Mathematics Letters, vol. 20, no. 9, pp. 1016-1022, 2007.

[11] L. Liu and Y. Bai, "New oscillation criteria for second-order nonlinear neutral delay differential equations," Journal of Computational and Applied Mathematics, vol. 231, no. 2, pp. 657-663, 2009.

[12] R. N. Rath, N. Misra, and L. N. Padhy, "Oscillatory and asymptotic behaviour of a nonlinear second order neutral differential equation," Mathematica Slovaca, vol. 57, no. 2, pp. 157-170, 2007.

[13] S. G. Ruan, "Oscillations of second order neutral differential equations," Canadian Mathematical Bulletin, vol. 36, no. 4, pp. 485-496, 1993.

[14] Y. G. Sun and F. Meng, "Note on the paper of Dourina and Stavroulakis," Applied Mathematics and Computation, vol. 174, no. 2, pp. 1634-1641, 2006. 
[15] Y. Şahiner, "On oscillation of second order neutral type delay differential equations," Applied Mathematics and Computation, vol. 150, no. 3, pp. 697-706, 2004.

[16] R. Xu and F. Meng, "Some new oscillation criteria for second order quasi-linear neutral delay differential equations," Applied Mathematics and Computation, vol. 182, no. 1, pp. 797-803, 2006.

[17] R. Xu and F. Meng, "Oscillation criteria for second order quasi-linear neutral delay differential equations," Applied Mathematics and Computation, vol. 192, no. 1, pp. 216-222, 2007.

[18] R. Xu and F. Meng, "New Kamenev-type oscillation criteria for second order neutral nonlinear differential equations," Applied Mathematics and Computation, vol. 188, no. 2, pp. 1364-1370, 2007.

[19] Z. Xu and X. Liu, "Philos-type oscillation criteria for Emden-Fowler neutral delay differential equations," Journal of Computational and Applied Mathematics, vol. 206, no. 2, pp. 1116-1126, 2007.

[20] L. Ye and Z. Xu, "Oscillation criteria for second order quasilinear neutral delay differential equations," Applied Mathematics and Computation, vol. 207, no. 2, pp. 388-396, 2009.

[21] A. Zafer, "Oscillation criteria for even order neutral differential equations," Applied Mathematics Letters, vol. 11, no. 3, pp. 21-25, 1998.

[22] Q. Zhang, J. Yan, and L. Gao, “Oscillation behavior of even-order nonlinear neutral differential equations with variable coefficients," Computers and Mathematics with Applications, vol. 59, no. 1, pp. $426-430,2010$.

[23] R.-K. Zhuang and W.-T. Li, "Interval oscillation criteria for second order neutral nonlinear differential equations," Applied Mathematics and Computation, vol. 157, no. 1, pp. 39-51, 2004. 